\title{
VARIAÇÃO ESPAÇO-TEMPORAL DOS PARÂMETROS PARA A MODELAGEM ESTOCÁSTTICA DA PRECIPITAÇÃO PLUVIAL DIÁRIA NO RIO GRANDE DO SUL ${ }^{1}$
}

\author{
MARCELO CALGARO ${ }^{2}$, ADROALDO D. ROBAINA ${ }^{3}$, MARCIA X. PEITER ${ }^{4}$, \\ TATIANE BERNARDON ${ }^{5}$
}

\begin{abstract}
RESUMO: O Rio Grande do Sul é um dos maiores produtores de grãos do Brasil, sendo a maioria das culturas conduzida em sistemas não irrigados, tornando-lhes extremamente dependentes das condições climáticas e do regime pluviométrico. Tal fato inspira diversas pesquisas na tentativa de caracterizar o comportamento espacial e temporal das precipitações. Dessa forma, o objetivo do presente trabalho foi estudar a variação espacial e temporal dos parâmetros alfa e beta da função gama para a geração de dados de precipitação pluvial diária no Estado. Os dados de precipitação pluviométrica foram obtidos junto à ANA - Agência Nacional de Águas. A modelagem da precipitação diária foi dividida em ocorrência e quantidade. Realizou-se a estimativa dos parâmetros alfa e beta da função gama a partir das séries históricas, seguida do ajuste desses parâmetros em função do tempo e do espaço e posterior comparação entre os valores estimados e os observados. De acordo com os resultados obtidos, conclui-se que a metodologia desenvolvida pode ser aplicada para a geração de séries sintéticas de precipitação pluvial diária nas diferentes estações pluviométricas do Rio Grande do Sul.
\end{abstract}

PALAVRAS-CHAVE: simulação, chuva, séries históricas.

\section{TEMPORAL AND SPATIAL VARIATION OF PARAMETERS FOR THE RANDOM MODELING OF DAILY RAINFALL IN THE STATE OF RIO GRANDE DO SUL, BRAZIL}

\begin{abstract}
Rio Grande do Sul is one of the largest grain producer states in Brazil, since most of the crops grown in non-irrigated systems, thus making them extremely dependent on the climatic conditions and on the pluviometric regime. This fact has inspired several research studies in the attempt of characterizing the spatial and temporal behavior of the rainfall. The objective of this research was to study the spatial and temporal variation of alpha and beta parameters of the gamma function for the generation of daily rainfall data in the state. The pluviometric precipitation data were obtained from the National Water Agency (ANA). The modeling of the daily rainfall was divided into the events of occurrence and quantity. Estimation of alpha and beta parameters of the gamma function was accomplished on the basis of historical series followed by the adjustment of these parameters as a function of time and space, and then the comparison between estimated and observed values. According to the results, one can conclude that the proposed methodology can be applied for the generation of synthetic series of daily rainfall in the different pluviometric stations of Rio Grande do Sul.
\end{abstract}

KEYWORDS: simulation, rainfall, time series.

\footnotetext{
${ }^{1}$ Extraído da tese de Doutorado do primeiro autor.

${ }^{2}$ Eng $^{\text {o }}$ Agrônomo, Doutor, Embrapa Semiárido, Petrolina - PE, Fone: (0XX87) 3862-1711, marcelo.calgaro@ cpatsa.embrapa.br

${ }^{3}$ Eng ${ }^{\mathrm{O}}$ Agrônomo, Doutor, Professor Titular, Departamento de Engenharia Rural, UFSM, Santa Maria - RS.

${ }^{4}$ Eng ${ }^{2}$ Agrônoma, Doutora, Professora Adjunta, Departamento de Engenharia Rural, UFSM, Santa Maria - RS.

${ }^{5}$ Bióloga, Mestre, Petrolina - PE.

Recebido pelo Conselho Editorial em: 12-9-2007

Aprovado pelo Conselho Editorial em: 1ํ-5-2009
} 


\section{INTRODUÇÃO}

O conhecimento da distribuição das precipitações pluviais no tempo e no espaço tem grande importância no planejamento das atividades agrícolas, e uma das maneiras de se conhecer essa distribuição é por meio da modelagem dessa variável no tempo e no espaço.

A modelagem da precipitação é de grande utilidade para diversos campos do conhecimento, destacando-se sua aplicação na área da Engenharia Agrícola, em que os modelos que consideram a chuva como um processo aleatório, e que são utilizados para simular sequências de chuvas mantendo as características estatísticas da série histórica, são conhecidos como modelos estocásticos. Um modelo estocástico é aquele que possui parâmetros obtidos a partir dos dados observados e que, apesar de não ser possível prever precisamente os valores que assumirá a precipitação no futuro, existe a possibilidade de fazer avaliações das probabilidades associadas aos valores futuros.

Em planejamento de sistemas irrigados, a modelagem da precipitação pluvial e a geração de séries sintéticas de distintos locais oferecem aos técnicos inúmeras possibilidades de avaliação do sistema. Dentre essas avaliações, pode-se destacar a análise da viabilidade econômica da implementação da irrigação em um dado local.

PEITER \& CHAUDHRY (2000), analisando a viabilidade econômica do uso de irrigação para a cultura do milho, propõem o uso de séries sintéticas de chuva como dados de entrada para modelo de simulação do crescimento dessa espécie. Com os resultados de produção a longo prazo, gerados pelo modelo de simulação, com base nas séries sintéticas de chuva, esses autores propõem metodologia que permita a análise técnica e econômica do uso de irrigação nessa cultura. De forma similar, FRIZZONE et al. (2005) realizam vasta revisão sobre trabalhos aplicados ao planejamento de sistemas irrigados que utilizam a modelagem estocástica da precipitação pluvial por meio das cadeias de Markov para a previsão da ocorrência e da distribuição gama para sua magnitude (HOWELL et al., 1975; AZEVEDO \& LEITÃO, 1990; ASSIS, 1991; FIETZ et al., 1998; PEITER, 1998).

Muito embora o procedimento para a simulação da precipitação pluvial seja conhecido e citado por diversos autores na literatura técnica, a variabilidade espacial e temporal dos parâmetros relacionados a esse procedimento é pouco analisada. Os procedimentos citados na literatura realizam avaliações locais sem possibilidade de extrapolação desses resultados para áreas maiores, como é o caso do Estado do Rio Grande do Sul. Sendo assim, o presente trabalho teve como objetivo estudar a variação espacial e temporal dos parâmetros para a modelagem da precipitação pluvial diária no Estado do Rio Grande do Sul.

\section{MATERIAL E MÉTODOS}

Os dados de precipitação pluvial diária utilizados para a realização deste trabalho foram obtidos junto à ANA - Agência Nacional de Águas. Foram escolhidas quatro estações com o maior número de anos completos para serem representativas dentro de cada região do Estado do Rio Grande do Sul (Tabela 1), com exceção da região da Lagoa dos Patos, totalizando 48 estações (quatro por região).

Os dados foram padronizados por meio da eliminação dos anos que não continham série completa dentro de cada arquivo, e a modelagem da precipitação diária foi dividida em duas etapas, em que, primeiramente, foi realizada a modelagem da ocorrência da precipitação e, posteriormente, da quantidade de precipitação (GENOVEZ, 1987; PEITER, 1998; NISHIJIMA, 2004), sendo a modelagem da ocorrência da precipitação pluvial diária utilizada neste trabalho, baseada em uma cadeia de Markov de primeira ordem. 
TABELA 1. Nome da estação pluviográfica, coordenadas e período em anos. Pluviographic station name, coordinates and period in years.

\begin{tabular}{|c|c|c|c|c|c|c|c|}
\hline Nome da Estação & Latitude & Longitude & $\begin{array}{c}\mathrm{N}^{\circ} \mathrm{de} \\
\text { anos }\end{array}$ & Nome da Estação & Latitude & Longitude & $\begin{array}{c}\mathrm{N}^{\circ} \mathrm{de} \\
\text { anos }\end{array}$ \\
\hline Erebango & -27.8542 & -52.3039 & 57 & Barros Cassal & -29.0850 & -52.5844 & 13 \\
\hline Itatiba do Sul & -27.3889 & -52.4544 & 26 & Candelária & -29.6733 & -52.7694 & 16 \\
\hline Linha Cescon & -27.8117 & -53.0278 & 41 & Serra dos Pedrosas & -30.6219 & -52.8097 & 23 \\
\hline Liberato Salzano & -27.5983 & -53.0708 & 27 & Pantano Grande & -30.1983 & -52.3719 & 17 \\
\hline Carazinho & -28.2939 & -52.7883 & 49 & Antonio Prado & -28.8533 & -51.2836 & 52 \\
\hline Conceição & -28.4550 & -53.9708 & 17 & Passo do Prata & -28.8675 & -51.4456 & 45 \\
\hline Santa Clara do Ingai & -28.7286 & -53.1858 & 42 & Prata & -28.7686 & -51.6200 & 57 \\
\hline Não Me Toque & -28.4553 & -52.8158 & 56 & Nova Palmira & -29.3347 & -51.1886 & 60 \\
\hline Paim Filho & -27.7039 & -51.7675 & 38 & $\overline{\text { Garruchos }}$ & -28.1825 & -55.6433 & 49 \\
\hline Sananduva & -27.9503 & -51.8144 & 45 & Itaqui & -29.1181 & -56.5578 & 33 \\
\hline Invernada Velha & -28.4494 & -50.2964 & 18 & Plano Alto & -29.7700 & -56.5158 & 55 \\
\hline Passo Socorro & -28.2108 & -50.7586 & 46 & Rosário do Sul & -30.2469 & -54.9175 & 55 \\
\hline Alto Uruguai & -27.3031 & -54.1344 & 51 & Dona Francisca & -29.6275 & -53.3533 & 56 \\
\hline Tucunduva & -27.6539 & -54.4422 & 27 & Tupanciretã & -29.0856 & -53.8219 & 25 \\
\hline Porto Lucena & -27.8544 & -55.0225 & 48 & Cacequi & -29.8778 & -54.8236 & 59 \\
\hline Giruá & -28.0264 & -54.3436 & 57 & Ernesto Alves & -29.3658 & -54.7347 & 43 \\
\hline Cerro Grande & -30.5981 & -51.7544 & 26 & Passo do Cação & -30.9611 & -53.4886 & 15 \\
\hline Guaiba Country Club & -30.1067 & -51.6489 & 29 & Dom Pedrito & -30.9781 & -54.6758 & 51 \\
\hline Quitéria & -30.4192 & -52.0733 & 32 & Torquato Severo & -31.0286 & -54.1844 & 25 \\
\hline Pacheca & -31.1303 & -51.7886 & 24 & Estância do Espantoso & -31.5314 & -54.2944 & 14 \\
\hline Glorinha & -29.8825 & -50.7883 & 22 & $\overline{\text { Canguçu }}$ & -31.3911 & -52.6972 & 48 \\
\hline Terra de Areia & -29.5722 & -50.0564 & 28 & Passo do Mendonça & -31.0003 & -52.0497 & 37 \\
\hline Sapucaia do Sul & -29.8211 & -51.4953 & 37 & Granja Santa Maria & -32.4044 & -52.5558 & 34 \\
\hline Palmares do Sul & -30.2531 & -50.5064 & 27 & Granja Osório & -32.9525 & -53.1189 & 29 \\
\hline
\end{tabular}

A metodologia para a modelagem da ocorrência e da quantidade de precipitação e quais parâmetros interferem na sua determinação, segundo PEITER (1998), podem ser vistos na Figura 1.

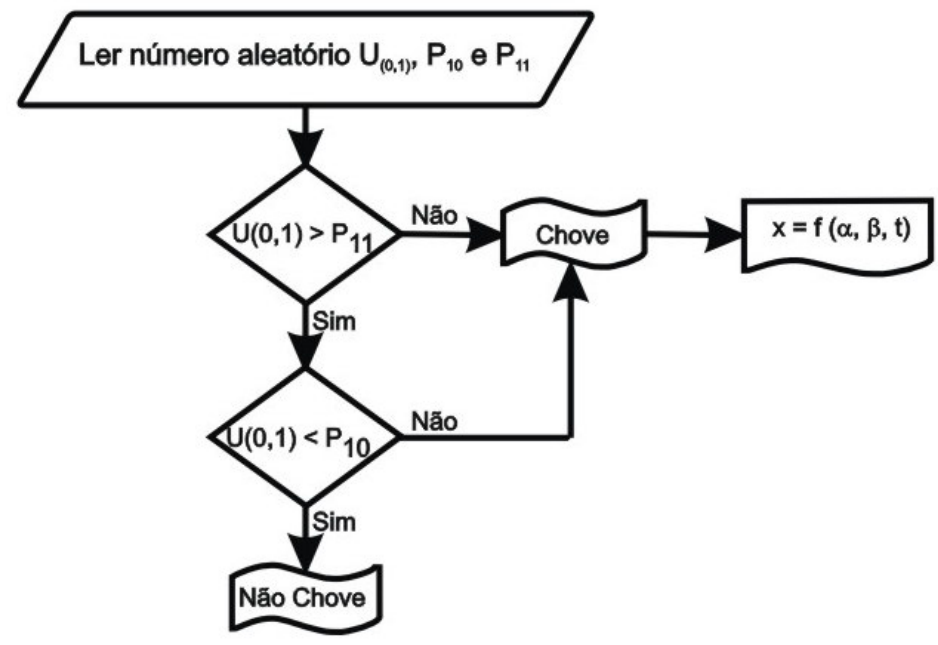

FIGURA 1. Fluxograma do processo de modelagem da precipitação pluvial (PEITER (1998), adaptado por CALGARO (2006)). Pluvial precipitation modeling process Flowchart (PEITER (1998), adapted by CALGARO (2006)). 
Para a definição da ocorrência de precipitação, foi determinada a matriz de transição (MT) em função das séries históricas disponíveis em cada local selecionado, a qual, neste trabalho, foi modificada para que seus elementos representassem a posição geográfica da estação, sendo:

$$
\operatorname{MT}(\varphi, \lambda, t)=\left[\begin{array}{ll}
\mathrm{P}_{00}(\varphi, \lambda, t) & \mathrm{P}_{01}(\varphi, \lambda, t) \\
\mathrm{P}_{10}(\varphi, \lambda, t) & \mathrm{P}_{11}(\varphi, \lambda, t)
\end{array}\right]
$$

em que,

MT - matriz de transição;

$\mathrm{P}_{00}$ - probabilidade de não chover hoje dado que não choveu ontem;

$\mathrm{P}_{01}$ - probabilidade de não chover hoje dado que choveu ontem;

$\mathrm{P}_{10}$ - probabilidade de chover hoje dado que não choveu ontem;

$\mathrm{P}_{11}$ - probabilidade de chover hoje dado que choveu ontem e onde $\mathrm{t}$ é a época do ano considerada;

$\phi$ e $\lambda$ - latitude e longitude do posto pluviométrico, e

$\mathrm{t}$ - época do ano considerada.

Foi considerado dia chuvoso, o dia em que a precipitação foi maior ou igual a $0,2 \mathrm{~mm}$.

A variação da probabilidade de ocorrência de precipitação $\mathrm{P}_{10}$ e a variação da probabilidade de ocorrência de precipitação $\mathrm{P}_{11}$, em função do tempo, foi ajustada para cada estação por modelo senoidal, conforme NISHIJIMA (2004):

$$
\begin{aligned}
& P_{10}(t)=a_{o}+a_{1} \operatorname{sen}(2 \theta)+a_{2} \cos (2 \theta)+a_{3} \operatorname{sen}(4 \theta)+a_{4} \cos (4 \theta) \\
& P_{11}(t)=b_{0}+b_{1} \operatorname{sen}(2 \theta)+b_{2} \cos (2 \theta)+b_{3} \operatorname{sen}(4 \theta)+b_{4} \cos (4 \theta)
\end{aligned}
$$

em que,

$a_{0}, a_{1}, a_{2}, a_{3}, a_{4}, b_{0}, b_{1}, b_{2}, b_{3}$ e $b_{4}$ - coeficientes determinados pelo método dos mínimos

$\theta=\pi t / 365, \mathrm{e}$ quadrados;

$\mathrm{t}$ - dia do ano.

Os coeficientes $a_{i}$ do elemento $\mathrm{P}_{10}(\mathrm{t})$ e os coeficientes $b_{i}$ do elemento $\mathrm{P}_{11}(\mathrm{t})$ da matriz de transição foram ajustados em função da posição geográfica $(\phi, \lambda)$ da estação por:

$$
\begin{aligned}
& a_{i}=c_{0 i}+c_{1 i} \phi+c_{2 i} \lambda \\
& b_{i}=c_{3 i}+c_{4 i} \phi+c_{5 i} \lambda
\end{aligned}
$$

em que,

$\mathrm{c}_{0 \mathrm{i}}, \mathrm{c}_{1 \mathrm{i}}, \mathrm{c}_{2 \mathrm{i}}, \mathrm{c}_{3 \mathrm{i}}, \mathrm{c}_{4 \mathrm{i}}$ e $\mathrm{c}_{5 \mathrm{i}}$ - coeficientes determinados pela técnica dos mínimos quadrados;

$\phi$ e $\lambda$ - latitude e longitude da estação, e

i - $0 ; 1 ; 2 ; 3$ e 4 .

Para a modelagem da quantidade de precipitação pluvial diária, foi adotada, conforme GENOVEZ (1987), PEITER (1998) e NISHIJIMA (2004), a distribuição de probabilidade gama de dois parâmetros cuja função de distribuição acumulada é:

$$
F_{X}(x)=\frac{1}{\Gamma(\alpha) \beta^{\alpha}} \int_{0}^{x} x^{\alpha-1} e^{\frac{-x}{\beta}} d x
$$

em que,

$\Gamma(\alpha)$ - função gama, e

$\alpha$ e $\beta$ - parâmetros da distribuição gama, sendo que a estimativa dos parâmetros $\alpha$ e $\beta$ foi feita pelo método da verossimilhança (THOM, 1966).

A variação do parâmetro $\alpha$ e a variação do parâmetro $\beta$ da função gama, em função do tempo (dia do ano), foi ajustada para cada estação por modelo senoidal: 


$$
\begin{aligned}
& \alpha(t)=c_{0}+c_{1} \operatorname{sen}(2 \theta)+c_{2} \cos (2 \theta)+c_{3} \operatorname{sen}(4 \theta)+c_{4} \cos (4 \theta) \\
& \beta(t)=d_{0}+d_{1} \operatorname{sen}(2 \theta)+d_{2} \cos (2 \theta)+d_{3} \operatorname{sen}(4 \theta)+d_{4} \cos (4 \theta)
\end{aligned}
$$

em que,

$\alpha(\mathrm{t})$ - parâmetro alfa e parâmetro beta da função gama em função do tempo;

$\mathrm{t}$ - dia do ano, e

$\mathrm{c}_{0}, \mathrm{c}_{1}, \mathrm{c}_{2}, \mathrm{c}_{3,}, \mathrm{c}_{4}, \mathrm{~d}_{0}, \mathrm{~d}_{1}, \mathrm{~d}_{2}, \mathrm{~d}_{3}$ e $\mathrm{d}_{4}$ - coeficientes da função harmônica.

Os coeficientes $c_{0}, c_{1}, c_{2}, c_{3}$ e $c_{4}$, do parâmetro $\alpha(t)$, e os coeficientes $d_{0}, d_{1}, d_{2}, d_{3}$ e $d_{4}$, do parâmetro $\beta(\mathrm{t})$, da função gama, neste trabalho, foram ajustados em função das coordenadas geográficas $(\phi, \lambda)$ por:

$$
\begin{aligned}
& c_{\mathrm{i}}=\mathrm{d}_{0 \mathrm{i}}+\mathrm{d}_{1 \mathrm{i}} \varphi+\mathrm{d}_{2 \mathrm{i}} \lambda \\
& \mathrm{d}_{\mathrm{i}}=\mathrm{d}_{3 \mathrm{i}}+\mathrm{d}_{4 \mathrm{i}} \varphi+\mathrm{d}_{5 \mathrm{i}} \lambda
\end{aligned}
$$

em que,

$\mathrm{d}_{0 \mathrm{i}}, \mathrm{d}_{1 \mathrm{i},}, \mathrm{d}_{2 \mathrm{i}}, \mathrm{d}_{3 \mathrm{i}}, \mathrm{d}_{4 \mathrm{i}}$ e $\mathrm{d}_{5 \mathrm{i}}$ - coeficientes determinados pela técnica dos mínimos quadrados;

$\phi$ e $\lambda$ - latitude e longitude da estação;

i - $0 ; 1 ; 2 ; 3$ e 4 .

A validação da modelagem dos elementos da matriz de transição e dos parâmetros da função gama, em função do tempo e do espaço, foi feita por meio da análise de regressão através do modelo linear $\mathrm{Y}=\mathrm{a}+\mathrm{b} \mathrm{X}$, em que $\mathrm{Y}$ são os valores observados e $\mathrm{X}$ são os valores estimados da variável em estudo $\left(\mathrm{P}_{10}, \mathrm{P}_{11}, \alpha\right.$ ou $\left.\beta\right)$. Para isso, foram utilizadas cinco estações-controle, representando os quatro pontos cardeais, mais uma representando o centro do Estado (Tabela 2).

TABELA 2. Região, município, estação, número de anos de dados e coordenadas geográficas. Region, city, station, data number of years and geographic coordinates.

\begin{tabular}{llllccc}
\hline $\mathrm{N}^{\circ}$ & Região & Município & Estação & $\mathrm{N}^{\circ}$ anos & Latitude & Longitude \\
\hline 1 & Norte & Miraguaí & Miraguaí & 25 & $-29^{\circ} 30^{\prime}$ & $-53^{\circ} 41^{\prime}$ \\
2 & Leste & Casca & Passo Migliavaca & 46 & $-28^{\circ} 37^{\prime}$ & $-51^{\circ} 52^{\prime}$ \\
3 & Centro & Júlio de Castilhos & Quevedos & 24 & $-29^{\circ} 51^{\prime}$ & $-54^{\circ} 04^{\prime}$ \\
4 & Oeste & Alegrete & Passo Mariano Pinto & 30 & $-29^{\circ} 18^{\prime}$ & $-56^{\circ} 03^{\prime}$ \\
5 & Sul & Pelotas & Granja São Pedro & 36 & $-31^{\circ} 40^{\prime}$ & $-52^{\circ} 11^{\prime}$ \\
\hline
\end{tabular}

O processo de avaliação da modelagem da ocorrência e da quantidade de precipitação foi realizado com valores de séries geradas e de séries históricas e podem ser vistos na Figura 2.

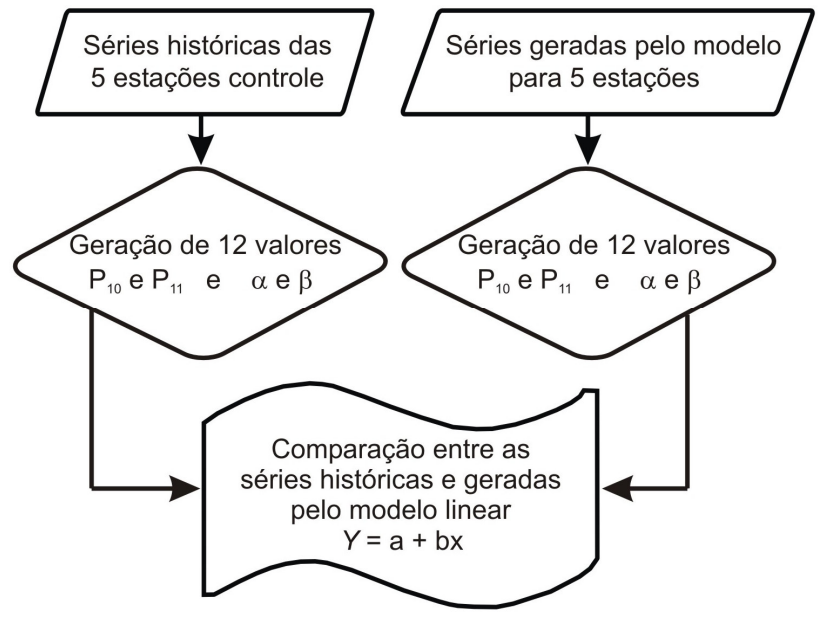

FIGURA 2. Fluxograma do processo de avaliação dos parâmetros obtidos com séries geradas. Flowchart of the evaluation process of the parameters obtained with generated series. 


\section{RESULTADOS E DISCUSSÃO}

A cadeia de Markov de primeira ordem foi utilizada em virtude dos resultados obtidos por vários autores, dentre os quais PEITER (1998) e NISHIJIMA (2004), que citam não haver necessidade de utilizar ordens mais elevadas para a modelagem da ocorrência de precipitação. A matriz de transição da ocorrência de precipitação foi modificada para que seus elementos representassem a posição geográfica da estação. Esse procedimento difere do adotado por GENOVEZ (1987), por PEITER (1998) e por NISHIJIMA (2004), em virtude da inclusão das coordenadas geográficas da estação a que os dados se referem.

\section{Ajuste do modelo dos elementos $P_{10}$ e $P_{11}$ da matriz de transição para as 12 regiões}

Os valores dos coeficientes do modelo da probabilidade de ocorrência $\mathrm{P}_{10}$, em função da média das coordenadas geográficas $(\phi, \lambda)$ das estações de cada região, podem ser vistos na Tabela 3, assim como os valores dos coeficientes do modelo da probabilidade de ocorrência $\mathrm{P}_{11}$ em função da média das coordenadas geográficas $(\phi, \lambda)$ das estações de cada região encontram-se na Tabela 4.

TABELA 3. Coeficientes do modelo da probabilidade de ocorrência $\mathrm{P}_{10}$, em função da média das coordenadas geográficas $(\phi, \lambda)$ das estações de cada região. Coefficients of the model of $P_{10}$ probability occurrence as a function of the geographic coordinates mean $(\phi, \lambda)$ of the stations of each region.

\begin{tabular}{crrrrrrr}
\hline Região & Latitude & Longitude & $\mathrm{a}_{0}$ & $\mathrm{a}_{1}$ & $\mathrm{a}_{2}$ & $\mathrm{a}_{3}$ & $\mathrm{a}_{4}$ \\
\hline 1 & $-27,6633$ & $-52,7142$ & 0,1919 & $-0,0053$ & 0,0285 & 0,0101 & $-0,0013$ \\
2 & $-28,4832$ & $-53,1902$ & 0,1742 & $-0,0031$ & 0,0185 & 0,0107 & $-0,0022$ \\
3 & $-28,0786$ & $-51,1592$ & 0,1804 & $-0,0067$ & 0,0276 & 0,0100 & $-0,0004$ \\
4 & $-27,7095$ & $-54,4857$ & 0,1818 & $-0,0029$ & 0,0212 & 0,0078 & $-0,0104$ \\
5 & $-30,5636$ & $-51,8163$ & 0,1766 & 0,0016 & 0,0086 & 0,0093 & 0,0012 \\
6 & $-29,8822$ & $-50,7116$ & 0,1772 & $-0,0036$ & 0,0110 & 0,0115 & 0,0011 \\
7 & $-29,9684$ & $-52,6339$ & 0,1812 & $-0,0003$ & 0,0155 & 0,0071 & $-0,0080$ \\
8 & $-28,9560$ & $-51,3845$ & 0,1978 & $-0,0023$ & 0,0226 & 0,0156 & 0,0000 \\
9 & $-29,3294$ & $-55,9086$ & 0,1528 & $-0,0001$ & 0,0090 & 0,0054 & $-0,0046$ \\
10 & $-29,4892$ & $-54,1834$ & 0,1697 & $-0,0058$ & 0,0055 & 0,0115 & $-0,0016$ \\
11 & $-31,1248$ & $-54,1608$ & 0,1462 & 0,0029 & 0,0065 & 0,0067 & $-0,0095$ \\
12 & $-31,9371$ & $-52,6054$ & 0,1306 & $-0,0016$ & 0,0039 & 0,0080 & $-0,0001$ \\
\hline Média & $-29,4321$ & $-52,9128$ & 0,1717 & $-0,0023$ & 0,0149 & 0,0095 & $-0,0030$ \\
Desvio-Padrão & 1,3477 & 1,5482 & 0,0193 & 0,0029 & 0,0087 & 0,0028 & 0,0041 \\
\hline
\end{tabular}

A análise dos valores dos coeficientes do modelo de probabilidade $\mathrm{P}_{10}$ e os seus respectivos desvios-padrão mostram a existência da variabilidade desses coeficientes em relação às diversas regiões estudadas, aproximando-se dos encontrados por MITCHELL (2000), que variaram entre 0,0079 e 0,0110 .

A comparação dos coeficientes da função harmônica de $\mathrm{P}_{10}$, em função do tempo, obtidos na Tabela 3, com os coeficientes obtidos por NISHIJIMA (2004), não foi possível devido ao fato de o autor ter utilizado os valores de $\mathrm{P}_{10}$ logaritmizados ao invés de utilizá-los diretamente, como foi realizado neste trabalho. Os valores encontrados por NISHIJIMA (2004) para $\mathrm{P}_{10}$ possuem média em torno de 0,22 e desvio-padrão em torno de 0,03, sendo semelhantes aos encontrados neste trabalho.

Pode-se observar que os valores médios dos coeficientes do modelo de probabilidade $\mathrm{P}_{11}$, da Tabela 4, e os seus respectivos desvios-padrão situaram-se dentro da faixa de valores $(0,0079$ e 0,0110) encontrados por MITCHELL (2000), mostrando a existência de pequena variabilidade entre esses coeficientes em relação às diversas estações. 
TABELA 4. Coeficientes do modelo da probabilidade de ocorrência $\mathrm{P}_{11}$, em função da média das coordenadas geográficas $(\phi, \lambda)$ das estações de cada região. Coefficients of the model of $P_{11}$ probability occurrence as a function of the geographic coordinates mean $(\phi, \lambda)$ of the stations of each region.

\begin{tabular}{crrrrrrr}
\hline Região & Latitude & Longitude & $\mathrm{b}_{0}$ & $\mathrm{~b}_{1}$ & $\mathrm{~b}_{2}$ & \multicolumn{1}{c}{$\mathrm{b}_{3}$} & \multicolumn{1}{c}{$\mathrm{b}_{4}$} \\
\hline 1 & $-27,6633$ & $-52,7142$ & 0,4313 & $-0,0052$ & $-0,0102$ & 0,0361 & 0,0005 \\
2 & $-28,4832$ & $-53,1902$ & 0,4277 & $-0,0018$ & $-0,0063$ & 0,0256 & 0,0016 \\
3 & $-28,0786$ & $-51,1592$ & 0,4419 & $-0,0023$ & 0,0185 & 0,0464 & 0,0114 \\
4 & $-27,7095$ & $-54,4857$ & 0,3933 & 0,0066 & $-0,0164$ & 0,0152 & $-0,0082$ \\
5 & $-30,5636$ & $-51,8163$ & 0,3906 & $-0,0038$ & $-0,0132$ & 0,0249 & $-0,0057$ \\
6 & $-29,8822$ & $-50,7116$ & 0,4314 & $-0,0126$ & $-0,0069$ & 0,0264 & $-0,0087$ \\
7 & $-29,9684$ & $-52,6339$ & 0,4117 & 0,0111 & $-0,0097$ & 0,0053 & $-0,0079$ \\
8 & $-28,9560$ & $-51,3845$ & 0,4299 & $-0,0054$ & 0,0008 & 0,0333 & 0,0184 \\
9 & $-29,3294$ & $-55,9086$ & 0,3150 & 0,0080 & $-0,0159$ & 0,0131 & $-0,0162$ \\
10 & $-29,4892$ & $-54,1834$ & 0,3620 & 0,0094 & $-0,0214$ & 0,0200 & $-0,0091$ \\
11 & $-31,1248$ & $-54,1608$ & 0,3503 & 0,0128 & $-0,0565$ & 0,0102 & $-0,0202$ \\
12 & $-31,9371$ & $-52,6054$ & 0,4156 & $-0,0015$ & $-0,0470$ & 0,0296 & $-0,0039$ \\
\hline Média & $-29,4321$ & $-52,9128$ & 0,4001 & 0,0013 & $-0,0153$ & 0,0238 & $-0,0040$ \\
Desvio-Padrão & 1,3477 & 1,5482 & 0,0394 & 0,0080 & 0,0199 & 0,0118 & 0,0108 \\
\hline
\end{tabular}

Ajuste dos coeficientes $\left(a_{0}, a_{1}, a_{2}, a_{3}\right.$ e $\left.a_{4}\right)$ do modelo harmônico de $P_{10}$ e dos coeficientes $\left(b_{0}, b_{1}\right.$, $b_{2}, b_{3}$ e $\left.b_{4}\right)$ do modelo harmônico de $P_{11}$, em função da média da posição geográfica $(\phi, \lambda)$ das estações

Os coeficientes da função de probabilidade de ocorrência de precipitação $\mathrm{P}_{10}(\phi, \lambda, t)$ foram relacionados por $\mathrm{a}_{\mathrm{i}}=\mathrm{c}_{0 \mathrm{i}}+\mathrm{c}_{1 \mathrm{i}} \phi+\mathrm{c}_{2 \mathrm{i}} \lambda$, e os coeficientes da função de probabilidade de ocorrência de precipitação $\mathrm{P}_{11}(\phi, \lambda, \mathrm{t})$ foram relacionados por $\mathrm{b}_{\mathrm{i}}=\mathrm{c}_{3 \mathrm{i}}+\mathrm{c}_{4 \mathrm{i}} \phi+\mathrm{c}_{5 \mathrm{i}} \lambda$, e podem ser observados na Tabela 5.

TABELA 5. Coeficientes de $a_{i}$ e de $b_{i}$ e soma dos quadrados dos resíduos. Coefficients of $\mathbf{a}_{\mathbf{i}}$ and $\mathbf{b}_{\mathbf{i}}$ and sum of residue squares.

\begin{tabular}{ccccc}
\hline Coeficientes & $\mathrm{c}_{0 \mathrm{i}}$ & $\mathrm{c}_{1 \mathrm{i}}$ & $\mathrm{c}_{2 \mathrm{i}}$ & $\mathrm{SQRes}$ \\
\hline $\mathrm{a}_{0}$ & 0,026827 & 0,003028 & $-0,004415$ & 0,004830 \\
$\mathrm{a}_{1}$ & 0,002597 & $-0,000651$ & 0,000453 & 0,000085 \\
$\mathrm{a}_{2}$ & 0,009216 & 0,002782 & $-0,001652$ & 0,000612 \\
$\mathrm{a}_{3}$ & 0,003602 & $-0,000229$ & 0,000017 & 0,000088 \\
$\mathrm{a}_{4}$ & 0,004820 & $-0,000948$ & 0,000676 & 0,000150 \\
\hline Coeficientes & $\mathrm{c}_{3 \mathrm{i}}$ & $\mathrm{c}_{4 \mathrm{i}}$ & $\mathrm{c}_{5 \mathrm{i}}$ & $\mathrm{SQRes}$ \\
\hline $\mathrm{b}_{0}$ & 0,064260 & $-0,008441$ & $-0,001635$ & 0,023334 \\
$\mathrm{~b}_{1}$ & 0,005633 & 0,001420 & $-0,000710$ & 0,000667 \\
$\mathrm{~b}_{2}$ & 0,020446 & 0,004513 & $-0,001826$ & 0,003515 \\
$\mathrm{~b}_{3}$ & 0,014590 & $-0,000535$ & 0,000126 & 0,001570 \\
$\mathrm{~b}_{4}$ & 0,012261 & 0,000293 & 0,000147 & 0,001217 \\
\hline
\end{tabular}

Conforme pode ser visto na Tabela 5, a soma dos quadrados dos resíduos resultantes da comparação entre os valores de $a_{i}$ (observados e estimados) variou entre 0,000088 e 0,004830, e a soma dos quadrados dos resíduos resultantes da comparação entre os valores de $b_{i}$ variou entre 0,001217 e 0,023334, indicando bom ajuste das grandezas envolvidas.

\section{Ajuste do parâmetro $\alpha$ e $\beta$ da função gama para as 12 regiões}

Os parâmetros $\alpha$ e $\beta$ da função gama são do tipo $\alpha=\alpha(\phi, \lambda, t)$ e $\beta=\beta(\phi, \lambda, t)$, em que $\phi$ é latitude, $\lambda$ é a longitude do posto pluviométrico e té a época do ano considerada. 
Na Tabela 6, são apresentados os coeficientes do modelo do parâmetro $\alpha$ da função gama, em função da média das coordenadas geográficas $(\phi, \lambda)$ das estações de cada região estudada.

TABELA 6. Coeficientes do parâmetro $\alpha$ da função gama, em função da média das coordenadas geográficas $(\phi, \lambda)$ das estações de cada região. $\alpha$ parameter coefficients of the gamma function, as a function of the $(\phi, \lambda)$ geographic coordinates mean of the stations of each region.

\begin{tabular}{crrrrrrr}
\hline Região & Latitude & Longitude & \multicolumn{1}{c}{$\mathrm{c}_{0}$} & \multicolumn{1}{c}{$\mathrm{c}_{1}$} & \multicolumn{1}{c}{$\mathrm{c}_{2}$} & \multicolumn{1}{c}{$\mathrm{c}_{3}$} & \multicolumn{1}{c}{$\mathrm{c}_{4}$} \\
\hline 1 & $-27,6633$ & $-52,7142$ & 1,2167 & 0,0162 & 0,0299 & 0,0385 & $-0,0158$ \\
2 & $-28,4832$ & $-53,1902$ & 1,2675 & $-0,0194$ & 0,0504 & 0,0313 & $-0,0119$ \\
3 & $-28,0786$ & $-51,1592$ & 1,2167 & 0,0162 & 0,0299 & 0,0385 & $-0,0158$ \\
4 & $-27,7095$ & $-54,4857$ & 1,1883 & $-0,0196$ & $-0,0213$ & 0,0391 & 0,0004 \\
5 & $-30,5636$ & $-51,8163$ & 1,2535 & $-0,0207$ & 0,0046 & 0,0318 & 0,0117 \\
6 & $-29,8822$ & $-50,7116$ & 1,2008 & $-0,0273$ & $-0,0012$ & 0,0173 & 0,0077 \\
7 & $-29,9684$ & $-52,6339$ & 1,1334 & $-0,0101$ & 0,0267 & $-0,0091$ & 0,0182 \\
8 & $-28,9560$ & $-51,3845$ & 1,3355 & 0,0057 & 0,0383 & 0,0406 & $-0,0180$ \\
9 & $-29,3294$ & $-55,9086$ & 1,1751 & $-0,0174$ & 0,0107 & 0,0237 & 0,0014 \\
10 & $-29,4892$ & $-54,1834$ & 1,2290 & 0,0001 & 0,0332 & 0,0219 & $-0,0020$ \\
11 & $-31,1248$ & $-54,1608$ & 1,1310 & $-0,0098$ & 0,1055 & 0,0077 & $-0,0092$ \\
12 & $-31,9371$ & $-52,6054$ & 1,1479 & $-0,0097$ & 0,0099 & $-0,0024$ & 0,0119 \\
\hline Média & $-29,4321$ & $-52,9128$ & 1,2079 & $-0,0080$ & 0,0264 & 0,0233 & $-0,0018$ \\
Desvio-Padrão & 1,3477 & 1,5482 & 0,0596 & 0,0145 & 0,0317 & 0,0169 & 0,0124 \\
\hline
\end{tabular}

Os valores médios dos coeficientes obtidos e seus respectivos desvios-padrão mostram a existência de pequena variação entre os valores dos coeficientes do modelo do parâmetro $\alpha$ da função gama, em função da média das coordenadas geográficas $(\phi, \lambda)$ das estações de cada região, fato esse que vem sendo observado para os demais fatores já apresentados.

Na Tabela 7, são apresentados os coeficientes do modelo do parâmetro $\beta$ da função gama, em função da média das coordenadas geográficas $(\phi, \lambda)$ das estações de cada região.

TABELA 7. Coeficientes do parâmetro $\beta$ da função gama, em função da média das coordenadas geográficas $(\phi, \lambda)$ das estações de cada região. $\beta$ parameter coefficients of the gamma function, as a function of the $(\phi, \lambda)$ geographic coordinates mean of the stations of each region.

\begin{tabular}{crrrrrrr}
\hline Região & Latitude & Longitude & \multicolumn{1}{c}{$\mathrm{d}_{\mathrm{o}}$} & \multicolumn{1}{c}{$\mathrm{d}_{1}$} & \multicolumn{1}{c}{$\mathrm{d}_{2}$} & \multicolumn{1}{c}{$\mathrm{d}_{3}$} & \multicolumn{1}{c}{$\mathrm{d}_{4}$} \\
\hline 1 & $-27,6633$ & $-52,7142$ & 16,1298 & 0,3090 & $-0,5664$ & $-1,2973$ & $-0,4644$ \\
2 & $-28,4832$ & $-53,1902$ & 14,9278 & $-0,9190$ & $-1,4505$ & $-0,3065$ & $-0,1939$ \\
3 & $-28,0786$ & $-51,1592$ & 15,7155 & 0,3088 & 0,2725 & $-1,5109$ & $-0,8120$ \\
4 & $-27,7095$ & $-54,4857$ & 13,4181 & 0,0047 & $-0,8668$ & $-0,6542$ & $-0,0945$ \\
5 & $-30,5636$ & $-51,8163$ & 12,8955 & 0,2162 & $-0,6657$ & 0,0559 & 0,2840 \\
6 & $-29,8822$ & $-50,7116$ & 16,2532 & $-0,6241$ & $-1,0858$ & $-0,0605$ & $-0,5943$ \\
7 & $-29,9684$ & $-52,6339$ & 12,5027 & $-0,6650$ & $-0,9952$ & $-0,0961$ & $-0,1444$ \\
8 & $-28,9560$ & $-51,3845$ & 12,5027 & 0,0745 & $-1,0382$ & $-1,5465$ & 0,0248 \\
9 & $-29,3294$ & $-55,9086$ & 17,5930 & 0,5476 & $-0,6625$ & $-1,4352$ & $-1,3669$ \\
10 & $-29,4892$ & $-54,1834$ & 17,7485 & 1,0803 & $-0,9519$ & $-1,7009$ & $-0,9769$ \\
11 & $-31,1248$ & $-54,1608$ & 14,5201 & 0,9024 & $-0,5445$ & 0,5116 & $-0,1649$ \\
12 & $-31,9371$ & $-52,6054$ & 16,0941 & 0,1909 & $-1,7076$ & $-1,4181$ & $-0,6216$ \\
\hline Média & $-29,4321$ & $-52,9128$ & 15,6649 & 0,3192 & $-0,6730$ & $-0,8152$ & $-0,6055$ \\
Desvio-Padrão & 1,3477 & 1,5482 & 2,2108 & 0,9121 & 0,7570 & 0,8120 & 0,6529 \\
\hline
\end{tabular}


Da mesma forma que o caso anterior, os valores médios dos coeficientes do modelo do parâmetro $\beta$ e os seus respectivos desvios-padrão mostram a existência de maior variabilidade desses coeficientes em relação às diversas estações analisadas.

Ajuste dos coeficientes $\left(a_{0}, a_{1}, a_{2}, a_{3}\right.$ e $\left.a_{4}\right)$ do parâmetro $\alpha$ e $\left(b_{0}, b_{1}, b_{2}, b_{3}\right.$ e $\left.b_{4}\right)$ do parâmetro $\beta$, em função da média da posição geográfica $(\phi, \lambda)$ das estações

Os coeficientes da função do parâmetro $\alpha$, em função da latitude $(\phi)$ e da longitude $(\lambda)$ de cada estação, foram relacionados por uma função do tipo $Y=c_{0}+c_{1} X_{1}+c_{2} X_{2}$ e $Y=c_{3}+c_{4} X_{1}+$ $\mathrm{c}_{5} \mathrm{X}_{2}$, em que $\mathrm{Y}$ são os valores dos coeficientes de $\alpha, \mathrm{X}_{1}$ a latitude e $\mathrm{X}_{2}$ a longitude de cada estação. Os valores de $\mathrm{c}_{0 \mathrm{i}}, \mathrm{c}_{1 \mathrm{i}}$ e $\mathrm{c}_{2 \mathrm{i}}$; e $\mathrm{c}_{3 \mathrm{i}}, \mathrm{c}_{4 \mathrm{i}}$ e $\mathrm{c}_{5 \mathrm{i}}$ foram determinados pelo método dos mínimos quadrados e podem ser vistos na Tabela 8 , na qual se pode observar que a soma dos quadrados dos resíduos resultantes da comparação entre os valores observados e os valores estimados variou entre 0,001454 e 0,071221, indicando bom ajuste das grandezas envolvidas.

TABELA 8. Coeficientes $\mathrm{c}_{0 \mathrm{i}}$, $\mathrm{ci}_{1}$ e $\mathrm{c}_{2 \mathrm{i}}$; e $\mathrm{c}_{3 \mathrm{i}} \mathrm{c}_{4 \mathrm{i}} \mathrm{c}_{5 \mathrm{i}}$ do modelo $\mathrm{Y}=\mathrm{c}_{0}+\mathrm{c}_{1} \mathrm{X}_{1}+\mathrm{c}_{2} \mathrm{X}_{2} ; \mathrm{Y}=\mathrm{c}_{3}+\mathrm{c}_{4} \mathrm{X}_{1}+$ $c_{5} X_{2}$ e soma dos quadrados dos resíduos de $\alpha$ e $\beta$. Coefficients $\mathbf{c}_{0 \mathbf{i}}$, $\mathbf{c i}_{\mathbf{1}}$ and $\mathbf{c}_{2 \mathbf{i}}$; and $c_{3 \mathrm{i}} c_{4 \mathrm{i}} c_{5 \mathrm{i}}$ of the model $Y=c_{0}+c_{1} X_{1}+c_{2} X_{2} ; Y=c_{3}+c_{4} X_{1}+c_{5} . X_{2}$ and sum of residue squares of $\alpha$ and $\beta$.

\begin{tabular}{crrrc}
\hline Coeficientes & $\mathrm{c}_{0 \mathrm{i}}$ & $\mathrm{c}_{1 \mathrm{i}}$ & $\mathrm{c}_{2 \mathrm{i}}$ & SQRes \\
\hline $\mathrm{a}_{0}$ & 0,005869 & $-0,004923$ & $-0,019978$ & 0,071221 \\
$\mathrm{a}_{1}$ & 0,000197 & $-0,000821$ & 0,000698 & 0,002366 \\
$\mathrm{a}_{2}$ & $-0,000469$ & $-0,002929$ & 0,001148 & 0,010967 \\
$\mathrm{a}_{3}$ & 0,000730 & 0,004789 & $-0,003047$ & 0,002309 \\
$\mathrm{a}_{4}$ & $-0,000393$ & $-0,003715$ & 0,002099 & 0,001454 \\
\hline Coeficientes & $\mathrm{c}_{3 \mathrm{i}}$ & $\mathrm{c}_{4 \mathrm{i}}$ & $\mathrm{c}_{5 \mathrm{i}}$ & SQRes \\
\hline $\mathrm{b}_{0}$ & $-5,8313$ & 0,0004 & $-0,3944$ & 3,3642 \\
$\mathrm{~b}_{1}$ & $-11,3001$ & $-0,0810$ & 0,1708 & 3,1896 \\
$\mathrm{~b}_{2}$ & 4,7710 & 0,1493 & 0,0233 & 2,2673 \\
$\mathrm{~b}_{3}$ & $-4,4395$ & $-0,1908$ & $-0,0371$ & 5,8902 \\
$\mathrm{~b}_{4}$ & 5,0648 & $-0,0195$ & 0,1146 & 2,0669 \\
\hline
\end{tabular}

Validação do modelo de previsão das probabilidades $P_{10}$ e $P_{11}$ da matriz de transição e dos parâmetros $\alpha$ e $\beta$ da função gama

Na Tabela 9, são apresentados os valores dos coeficientes da regressão de ajuste das probabilidades de ocorrência de chuva $\mathrm{P}_{10}$ e $\mathrm{P}_{11}$ e dos parâmetros $\alpha$ e $\beta$. Pode-se observar que os valores estimados de $\alpha$ são estatisticamente iguais aos observados de $\alpha$ e que os valores estimados de $\mathrm{P}_{10}, \mathrm{P}_{11}$ e $\beta$ são estatisticamente diferentes dos observados de $\mathrm{P}_{10}, \mathrm{P}_{11}$ e $\beta$

TABELA 9. Valores dos coeficientes linear, angular e de determinação, e valores do teste do coeficiente b (t calculado e $t$ tabelado). Linear, angular, and determination coefficient values, and test values of the $b$ coefficient ( $t$ calculated and $t$ tabulated).

\begin{tabular}{ccccrrr}
\hline & $\mathrm{a}$ & $\mathrm{b}$ & \multicolumn{1}{c}{$\mathrm{r}^{2}$} & tb calc. & tb (95\%) & $\mathrm{S}$ ou NS \\
\hline $\mathrm{P}_{10}$ & 0,028 & 0,935 & 0,9477 & 7,06 & 2,01 & $\mathrm{~S}$ \\
$\mathrm{P}_{11}$ & 0,047 & 0,756 & 0,9614 & 38,55 & 2,01 & $\mathrm{~S}$ \\
\hline$\alpha$ & 0,091 & 0,999 & 0,9826 & 0,02 & 2,01 & $\mathrm{NS}$ \\
$\beta$ & $-0,319$ & 1,046 & 0,9686 & 5,89 & 2,01 & $\mathrm{~S}$ \\
\hline
\end{tabular}


Por meio da Tabela 9, percebe-se que os valores de $\mathrm{t}$ calculados foram significativos para $\mathrm{P}_{10}$, indicando que os valores estimados não são iguais aos valores observados; porém, se observadas as diferenças entre os coeficientes da regressão $(6,5 \%)$, nota-se que essas foram pequenas. Devido à diferença ser significativa, os resultados da probabilidade $\mathrm{P}_{10}$ obtidos com modelo proposto devem ser ajustados pela equação de regressão.

De maneira semelhante, pode-se perceber, na Tabela 9, que os valores de $t$ calculados foram significativos para $\mathrm{P}_{11}$, indicando que os valores observados de $\mathrm{P}_{11}$ não são iguais aos valores estimados, inclusive com diferença em torno de $24,4 \%$, que é maior que a encontrada para $\mathrm{P}_{10}$. Da mesma forma, como a diferença foi significativa, os resultados da probabilidade $\mathrm{P}_{11}$ obtidos com modelo proposto devem ser ajustados pela equação de regressão.

\section{Avaliação de $\mathbf{P}_{10}$ e $\mathbf{P}_{11}$, e $\alpha$ e $\beta$ obtidos com séries pluviométricas diárias geradas}

$\mathrm{Na}$ Tabela 10, são mostrados os valores dos coeficientes linear, angular e de determinação, bem como dos valores de $t$ calculados e tabelados, e a significância para as distribuições $P_{10}$ e $P_{11}$.

TABELA 10. Valores dos coeficientes linear, angular e de determinação, valores do teste $t$ calculados e tabelados, e nível de significância de $\mathrm{P}_{10}$ e $\mathrm{P}_{11}, \alpha$ e $\beta$. Linear, angular, and determination coefficient values, $t$ test calculated and tabulated values and significance level of $P_{10}$ and $P_{11}, \alpha$ and $\beta$.

\begin{tabular}{lcccccc}
\hline & $\mathrm{a}$ & $\mathrm{b}$ & $\mathrm{r}^{2}$ & $\mathrm{t}(\mathrm{b})$ calc. & $\mathrm{t}(\mathrm{b})$ tab. & $\mathrm{S}$ ou NS \\
\hline $\mathrm{P}_{10}$ e $\mathrm{P}_{11}$ & $\approx 0$ & 0,9628 & 0,9828 & 4,04 & 1,96 & $\mathrm{~S}$ \\
$\alpha$ e $\beta$ & $\approx 0$ & 1,0136 & 0,9976 & 2,98 & 1,96 & $\mathrm{~S}$ \\
\hline
\end{tabular}

$\mathrm{Na}$ análise da regressão ajustada entre as séries geradas e as séries históricas apresentadas na Tabela 10, observa-se que os valores de $\mathrm{t}$ tabelados são inferiores aos valores de $\mathrm{t}$ calculados, indicando, assim, que os valores de $\mathrm{P}_{10}$ e $\mathrm{P}_{11}$ das séries geradas não são estatisticamente iguais aos valores de $\mathrm{P}_{10}$ e $\mathrm{P}_{11}$ das séries históricas. Entretanto, pode-se perceber que os valores do coeficiente $\mathrm{b}$ da regressão ajustada são bem próximos a 1, apresentando diferença de apenas 3,7\% para $\mathrm{P}_{10} \mathrm{e}$ $\mathrm{P}_{11}$, podendo-se, portanto, fazer as correções dos parâmetros por esse valor e, dessa forma, utilizálos para gerar as séries de dados de precipitação.

Em relação aos valores de $\alpha$ e $\beta$ das séries geradas comparado com as séries históricas, pode-se observar, pelos valores de t (b), que não houve bom ajuste entre os valores, indicando, assim, que tais valores não são estatisticamente iguais; entretanto, pode-se perceber que os valores do coeficiente $b$ da regressão ajustada são bem próximos a 1 , tendo diferença de apenas $1,4 \%$, podendo-se, por meio desse, fazer as correções dos valores e, dessa forma, utilizá-los para gerar as séries de dados de precipitação. Nos dois casos, o que se deseja é que tais valores permaneçam em torno de 1, o que indicaria, conforme citado por COSTA NETO (1977), bom ajuste entre os dados.

\section{CONCLUSÕES}

De acordo com a metodologia proposta e os resultados obtidos, pode-se concluir que os elementos da matriz de transição e os parâmetros alfa e beta apresentam variabilidade em relação ao tempo e à posição geográfica da estação. No entanto, considerando-se os ajustes citados, é possível a utilização do modelo proposto para a geração de séries sintéticas em análise de sistemas que requeiram espacialização da precipitação pluvial como componente para o balanço hídrico para a região de estudo.

Pode-se também concluir que o procedimento metodológico proposto, que se caracterizou pela representação e pela obtenção dos parâmetros necessários a partir das coordenadas geográficas das estações de maneira analítica, é viável como modelo estocástico para uso no planejamento de recursos hídricos, considerando-se sistemas que demandem séries de precipitação pluvial. 


\section{REFERÊNCIAS}

ASSIS, F.N. Modelagem da ocorrência e da quantidade de chuva e de dias secos em PiracicabaSP e Pelotas - RS. 1991. 134 f. Tese (Doutorado em Física do Ambiente Agrícola) - Escola Superior de Agricultura "Luiz de Queiroz", Universidade de São Paulo, Piracicaba, 1991.

AZEVEDO, P.V.; LEITÃO, M.M.V.B.R. Aplicação de processos em cadeia de Markov às precipitações diárias do Estado da Paraíba. Revista Brasileira de Meteorologia, Santa Maria, v.5, n.1, p.389-402, 1990.

CALGARO, M. Análise da variação espaço-temporal dos parâmetros para a modelagem estocástica da precipitação pluvial do Rio Grande do Sul. 2006. 127 f. Tese (Doutorado em Engenharia de Água e Solo) - Universidade Federal de Santa Maria, Santa Maria, 2006.

COSTA NETO, P. L. Estatística. São Paulo: Edgard Blucher, 1977. 264 p.

FIETZ, C.R.; URCHEI, M.A.; FRIZZONE, J.A.; FOLEGATTI, M.V. Probabilidade de ocorrência de períodos secos e chuvosos na região de Dourados - MS. Irriga, Botucatu, v.3, n.1, p.16-22, 1998.

FRIZZONE, J.A.; ANDRADE JÚNIOR, A.S.; SOUZA, J.L.M.; ZOCOLER, J.L. Planejamento da irrigação. Brasília: EMBRAPA, 2005. 627 p.

GENOVEZ, A.M. Avaliação da regularização de vazões em pequenas bacias com dados esparsos. 1987. 168 f. Tese (Doutorado em Engenharia Civil) - Universidade de São Paulo, Escola de Engenharia de São Carlos, São Carlos, 1987.

HOWELL, T.A.; HILER, E.A.; REDDELL, D.L. Optimization of water use efficiency under high frequency irrigation. II. System simulation and dynamic programming. Transactions of the ASAE, St. Joseph, v.18, n.5, p.879-887, 1975.

MITCHELL, P.D. Generation of simulated daily precipitation and air and soil temperatures. Ames: Iowa State University, 2000. 33 p. (Working Paper 00-WP 234).

NISHIJIMA, T. Modelagem Markoviana da precipitação pluvial diária e simulação do rendimento esperado de soja no município de Cruz Alta - RS. 2004. 135 f. Tese (Doutorado em Recursos Hídricos e Saneamento Ambiental) - Universidade Federal do Rio Grande do Sul, Porto Alegre, 2004.

PEITER, M.X.; CHAUDHRY, F.H. Supplemental irrigation control strategies for corn by simulation of the management cycle. In: Fourth Decennial National Irrigation Symposium, 2000, Phoenix. Proceedings... Arizona: American Society of Agricultural Engineers, 2000. v.1, p.623628.

PEITER, M.X. Estudo do manejo da irrigação via modelo de simulação. 1998. 183 f. Tese (Doutorado em Hidráulica e Saneamento) - Universidade de São Paulo, Escola de Engenharia de São Carlos, São Carlos, 1998.

THOM, H.C.S. Some methods of climatological analysis. Roma: FAO, 1966. 50 p. (Technical Notes, 81). 